\title{
Calculation of 220kV Transformer Bias Current Based on the Earth and Power Grid Model in the Metro Area
}

\author{
Lu Chen ${ }^{1}$, Longfei Wang ${ }^{2}$, Hua Huang ${ }^{1}$, Chunming Liur,", Tianyi $\mathrm{Wu}^{1}$, Lei Su${ }^{1}$, and Dandan Zhao ${ }^{1}$ \\ ${ }^{1}$ State Grid Shanghai Municipal Electric Power Company, 1122 Yuanshen Road, Pudong New Area, Shanghai, China \\ ${ }^{2}$ North China Electric Power University, No. 2 Beinong Road, Changping, Beijing, China
}

\begin{abstract}
The metro stray current will be generated during subway operation, which will affect the electromagnetic environment near the subway, and then affect the surrounding $220 \mathrm{kV}$ transformer. In order to analyze the influence of stray current on the DC bias of the transformer, this paper calculates the magnitude of the stray current through the resistance network model, and then analyzes the changes in the surrounding ground potential caused by the stray current based on the earth resistivity model. Finally, the $220 \mathrm{kV}$ transformer network topology model of the city network was established, and the relationship between the neutral point current of the transformer and time was obtained. It provided a quantitative method for research the correlation between subway stray current and $220 \mathrm{kV}$ transformer bias current phenomenon and calculate the bias current of $220 \mathrm{kV}$ transformer in urban network near subway.
\end{abstract}

\section{Introduction}

With the continuous development of urban construction, the scale of my country's urban rail transit has become larger and larger, which has become the most convenient solution for citizens to travel. However, because urban subways or light rails mostly use DC traction power supply, the rails are not completely insulated from the ground. During the operation of subway trains, direct current will leak from the subway rails into the ground, forming the so-called the subway metro stray current. The DC metro stray current will flow into the transformer windings through the grounding grid of the urban power grid substation, causing saturation of the DC bias of the transformer, resulting in increased vibration and noise of the main transformer [1], and the temperature rise of the transformer core and clamps [2], Severe DC bias will cause damage to the transformer [3]. The influence of DC metro stray current city network $220 \mathrm{kV}$ transformer bias is a subject to be studied. In 1971, Boden P J et al. proposed the harmful effect of DC metro stray current on the corrosion of underground pipelines [4]. Literature [5] modeled the subway metro stray current on the Pspice platform, and calculated the stray current under unilateral and bilateral power supply modes. Literature [6] summarized the shortcomings of the circuit network component model and the neural network model, and proposed a stray current model based on the electric field. Literature [7] established a static distribution model of stray current for unilateral power supply and bilateral power supply. These studies are mainly to explore the impact of subway stray current on underground metal pipelines or other metal facilities. Literature [8] carried out a simple modeling on the coupling of stray current to the power grid within $1 \mathrm{~km}$ on the CDEGS platform. At present, there is no research literature report on establishing the ground and power grid model in the vicinity of the subway and calculating the bias current of $220 \mathrm{kV}$ transformer in the city grid.

\section{Earth potential model near the subway}

\subsection{Train leakage current model}

The model of train leakage current adopts a three-layer resistance network model [9], and the subway system adopts DC1500V power supply and double-sided power supply of the overhead catenary network, and the rail return. In order to reduce stray current leakage, measures such as setting gaskets and insulating rubber are adopted to increase the rail-to-ground transition resistance; At the same time, a drainage network is set in the concrete under the track to recover stray current. Therefore, the stray current flow path of the subway is to leak out from the track at the wheels of the train, enter the ground through the track bed structure and current drainage net, and flow back to the substation from the ground. According to the stray current leakage path, considering the parallel distribution of the pipeline and the track, the track, drain net, and ground are simplified into stacked resistors, and the different layers are connected by transition resistors. The stray current flow path can be regarded as a current loop of rail- current drainage net earth in parallel. The distribution of the established three-layer resistor network model is shown in Figure 1.In the figure, $\mathrm{I} 1$ and $\mathrm{I} 2$ are the magnitude of the current

\footnotetext{
*Corresponding author: cm_liu@163.com
} 
flowing through the train equivalent resistance, I 3 and I4 are the magnitude of the current flowing through the train track, I5 and I6 are the magnitude of the current flowing through the current drainage net, I7 and I8 are the magnitude of the leakage current .Since subway lines, drainage networks, and earth resistance are all uniform transmission line models, the circuit model should be differentiated, and the micro-element model of the circuit can be obtained as shown in Figure 2.

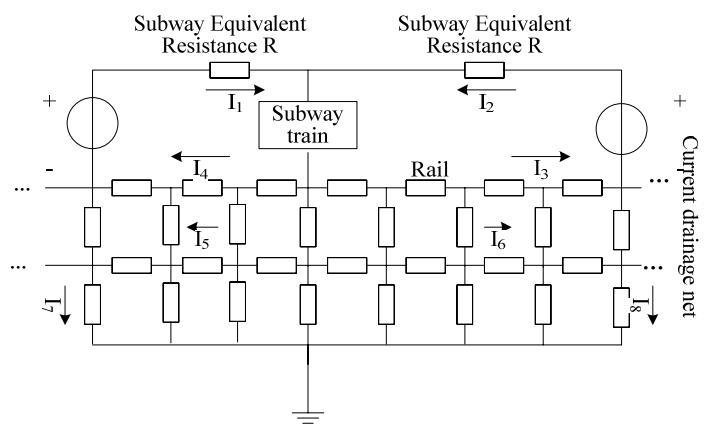

Figure 1. Circuit model of bilateral power supply mode

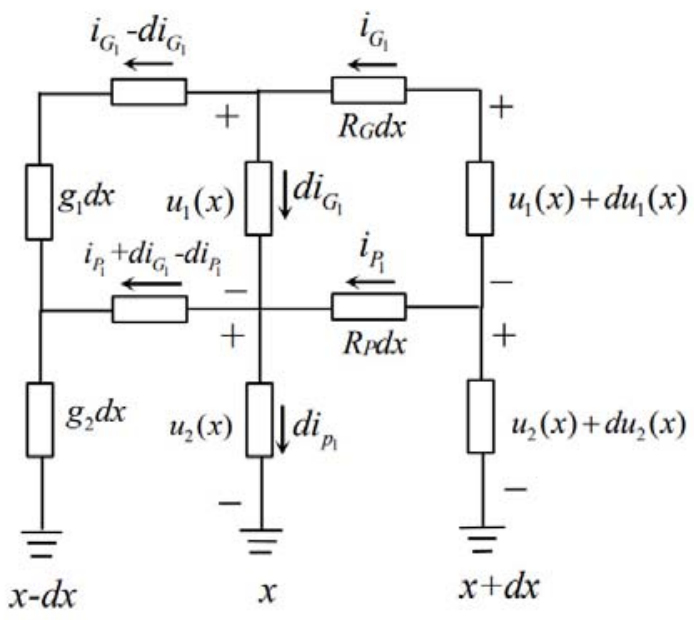

Figure 2. Circuit micro-element model

According to this model, KCL (2-1) and KVL (2-2) equations can be obtained:

$$
\begin{gathered}
\left\{\begin{array}{l}
\mathrm{d} i_{G_{1}}=u_{1}(x) g_{1} \mathrm{~d} x \\
\mathrm{~d} i_{P_{1}}=u_{2}(x) g_{2} \mathrm{~d} x
\end{array}\right. \\
\left\{\begin{array}{l}
i_{G_{1}} R_{G} \mathrm{~d} x+u_{1}(x)=i_{P_{1}} R_{P} \mathrm{~d} x+u_{1}(x)+\mathrm{d} u_{1}(x) \\
i_{P_{1}} R_{P} \mathrm{~d} x+u_{2}(x)=u_{2}(x)+\mathrm{d} u_{2}(x)
\end{array}\right.
\end{gathered}
$$

Solving the differential equation can get the total stray current when the train travels to a certain position. Set the train travel distance from 0 to $\mathrm{L}$ to get the stray current when the train travels on the whole line.

Assuming that the total stray current generated when the train travels to (1) x meters from the substation is Isc, which is defined by the stray current:

$$
I_{s c}=\sum\left(i_{s c 1}+i_{s c 2}\right)=I-I_{G}-I_{p}
$$

\subsection{Earth potential model}

In this section, based on the stray current model in the previous section, as well as the earth's electrical properties and its structural data and data, a meticulous earth resistivity model is established to calculate the ground potential of the substation around the subway. It should be noted that the location of subway trains is constantly changing, therefore, if the subway is running multiple trains at the position of turning, intersection, parallel, etc., the ground potential generated by the stray current generated by these trains can be overlay by Superposition theorem. The ground potential of the train can be obtained dynamically. In actual operation, there may be many trains running at the same time, and it is necessary to consider the changes in potential and time of the substation location.

When the subway is operating normally, assuming that a current of $i(t)$ will flow into the ground, the stray current into the ground is regarded as a driving current source, and the problem of solving the point distribution can be converted into a mathematical description of the current field. Specifically, based on Maxwell's equations, the field equations are derived, and the field equations near the rails are solved to express the law of earth potential distribution. First derive the field equations from the Maxwell equations, the basic form of the differential equation of the current field can be obtained as:

$$
\begin{gathered}
\nabla \times \vec{E}=0 \\
\nabla \bullet \vec{J}=-\frac{\partial \rho_{v}}{\partial t}
\end{gathered}
$$

Its auxiliary equation:

$$
\begin{aligned}
\vec{J} & =\sigma \vec{E} \\
\vec{E} & =-\nabla U
\end{aligned}
$$

Among them $E$ :Electric field strength $(\mathrm{V} / \mathrm{m})$

$J$ : Current density $\left(\mathrm{A} / \mathrm{m}^{2}\right)$

$\rho_{v}:$ Volume charge density $\left(\mathrm{C} / \mathrm{m}^{3}\right)$

$\sigma:$ Conductivity $(\mathrm{S} / \mathrm{m})$

$U$ : electric scalar potential(V)

There are two types of equations to describe the current field by using the field quantity $U$ to be calculated, they are Laplace equation without current source (2-8) and Poisson equation with current source(29).

$$
\begin{gathered}
\nabla^{2} U=0 \\
\nabla^{2} U=-\frac{\partial \rho_{v}}{\partial t}
\end{gathered}
$$

Since the electric field equation is unique, the electric field can be determined as long as the boundary conditions of the field are determined, and a unique solution can be obtained. Therefore, find out the boundary conditions of the electric field equation near the subway track, and use the finite element method to solve this equation to get the potential change distribution near the subway track. 


\subsection{Power grid DC bias current model}

The change of ground potential will generate a potential difference between the two grounds of the transformer, and then generate a bias current in the power grid. Based on GIC-Benchmark [11] and the topology of the power grid, the bias current model of the power grid is established.

When the subway is operating normally, the bias current in the actual power grid is complicated, and there are many factors that affect the bias current of the power grid. Consider the influence of these factors, simplify processing of grid data and parameters according to GIC-Benchmark. The simplified substation and power supply line bias current equivalent current is shown in Figure 3. Among them, $U_{n}(n=1,2, \ldots, n)$ is equivalent to acting on the neutral point potential of the two substation transformers, $R_{n}(n=1,2, \ldots, n)$ is the equivalent resistance of the circuit.

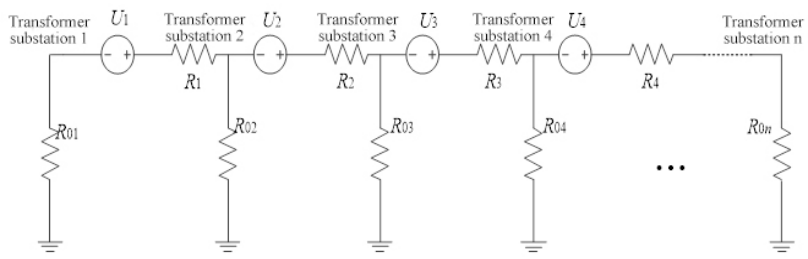

Figure 3. Calculate the equivalent circuit of the grid bias current

The node voltage method can be used to calculate the bias current of the grid, which is

$$
\left\{\begin{array}{c}
u_{1}=R_{01} I_{0}+R_{11} I_{1}+R_{12} I_{2}+\ldots+R_{1 n} I_{n} \\
u_{2}=R_{02} I_{0}+R_{21} I_{1}+R_{22} I_{2}+\ldots+R_{2 n} I_{n} \\
\vdots \\
u_{n}=R_{0 n} I_{0}+R_{n 1} I_{1}+R_{n 2} I_{2}+\ldots+R_{n n} I_{n}
\end{array}\right.
$$

Where: $n$ is the number of nodes, $I_{0}$ is the distribution value of the ground current in each power station, $R_{i i}$ is the self-admittance of point $i, R_{i j}$ is the mutual admittance between point $i$ and point $j, u_{i}$ and $I_{i}$ are the potential and current of the ground point $i$.

\section{Calculation examples and analysis}

\subsection{Modeling data}

A typical subway geographic map of a city is shown in Figure 4, and the data for establishing a model of the earth and power grid is shown in Table 1. According to Figure 4 and Table 1 data and information. Using the above algorithm of ground potential and grid bias current, establish the ground potential model and grid bias current model of the subway near area and considering the influence of factors such as train speed and number of trains, MATLAB programming is used to calculate the bias current at the neutral point of the $220 \mathrm{kV}$ transformer in the city network. In order to improve the calculation accuracy, the calculation micro-element is $1 \mathrm{~m}$ in the simulation calculation, the total length of the simulation line is $2000 \mathrm{~m}$, and there are a total of 2000 calculation micro-element. Related simulation parameter settings are shown in Table 1.

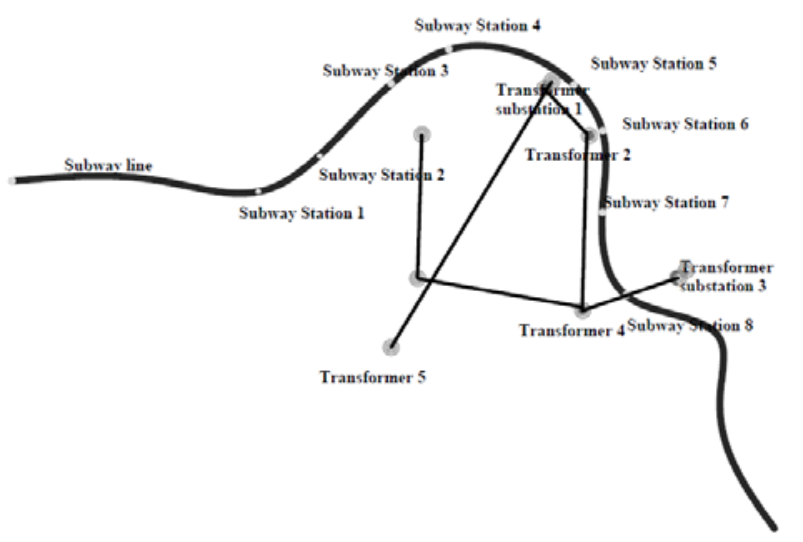

Figure 4. Geographical map of a city's subway system

Table 3. Font styles for a reference to a journal article.

\begin{tabular}{|c|c|}
\hline Term & Resistance( $\mathbf{\Omega})$ \\
\hline 60 Track & 0.00003436 \\
\hline $\begin{array}{c}\text { The Current } \\
\text { drainage network } \\
\text { resistance }\end{array}$ & 0.00001 \\
\hline $\begin{array}{c}\text { Rail to current } \\
\text { Drainage Network } \\
\text { Resistance }\end{array}$ \\
\hline $\begin{array}{c}\text { 15m thickness } \\
\text { Quaternary earth } \\
\text { surface resistance }\end{array}$ & $30 \times 15$ \\
\hline $\begin{array}{c}5 \mathrm{~m} \text { thick limestone } \\
\text { resistance }\end{array}$ & $700 \times 5$ \\
\hline
\end{tabular}

\subsection{Metro stray current and ground potential calculation}

The simulation results in Fig. 4 shows that the stray current between subway stations along the subway is shown in Fig. 5, and the ground potential change along the subway is shown in Fig. 6. In the calculation, the uniform earth resistance data used is $200 \Omega / \mathrm{m}$. It is assumed that the train is sent out from the departure station every five minutes according to the operation schedule, the train running time between stations is $2 \mathrm{~min}$, and the running mode is the first $40 \mathrm{~s}$ as the acceleration time. The last $40 \mathrm{~s}$ is the deceleration time, and it is moving at a constant speed during the middle operation. 


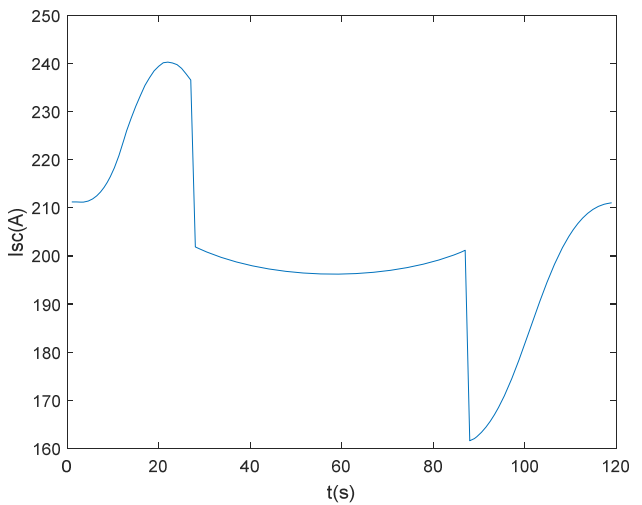

Figure 5. The curve of the stray current of the whole line with time

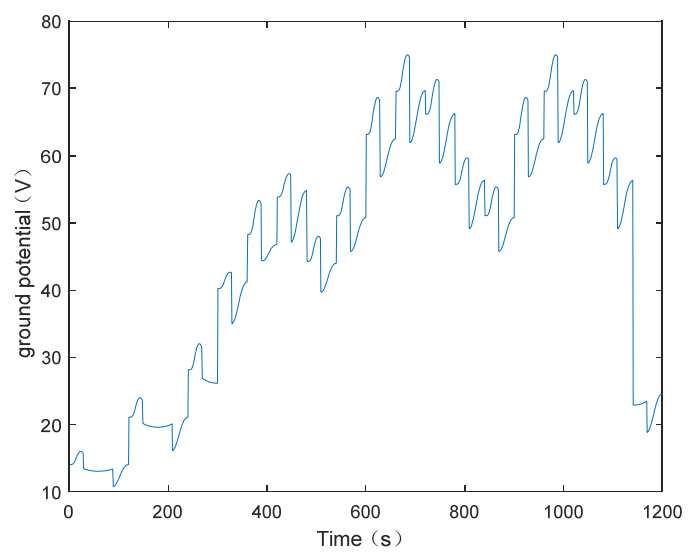

Figure 6. Change curve of ground potential of transformer substation 1 with time

\subsection{Power grid bias current calculation}

According to the calculated ground potential along the subway and the established bias current model of the urban power grid, the ground potential of each substation of the power grid and the potential difference between the ground points of the transformers of the two substations can be calculated, and then the two ground potentials that change with the ground potential can be calculated. The magnitude of the DC bias current at the neutral point of the transformer in the substation. The results obtained are shown in Figure 7 and Figure 8.

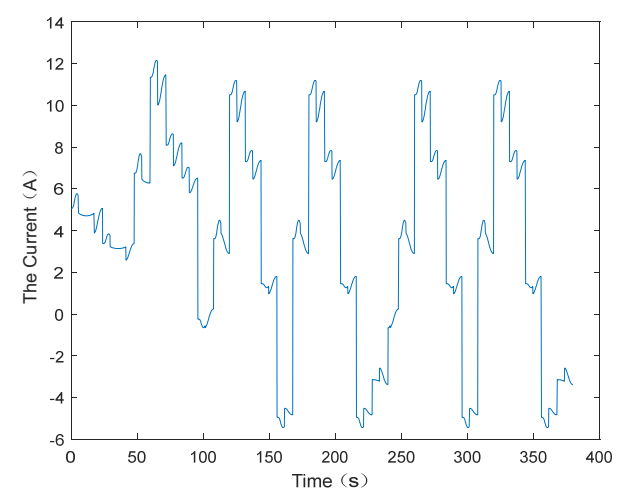

Figure 7. The bias current of the neutral point of transformer 1 in the substation

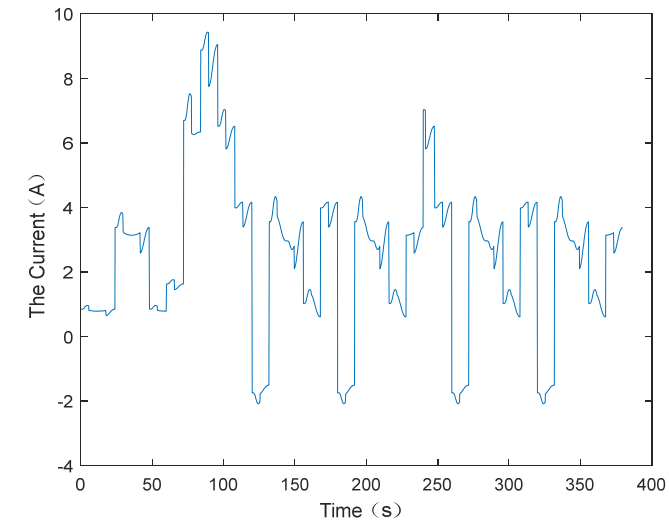

Figure 8. The bias current of the neutral point of transformer 2 in the substation

\section{Conclusion}

This paper adopts the three-layer physical model of "Metro-Earth-Grid" to complete the theoretical calculation of the influence of the leakage stray current of the subway system on the DC bias current of the transformer in the urban grid. The main research conclusions obtained are as follows:

1. Among the two substations, one substation transformer has a maximum bias current of $12.8 \mathrm{~A}$, the average value within one minute has reached $7.58 \mathrm{~A}$, and the maximum reverse bias current is $5.68 \mathrm{~A}$, and it appears periodically. Combined with the regulation of transformer operation, if it exceeds $3 \mathrm{~A}$, it needs to be treated, and measures should be taken to treat the transformer bias caused by the subway.

2. In a substation within the influence area of the same subway, if the number of feeders intervening in the substation and the line of defense connected to the substation are different, the DC bias current at the neutral point of the transformer in the substation will be different. You can know the magnitude of the grid bias current. The change trend is not only related to the operation mode of the subway, but also related to the topology of the power grid.

3. Because subway trains run fast, there are many trains on the same subway line, which will cause rapid changes in ground potential in a large area, so the bias current of the transformer can change directions multiple times within one minute.

\section{Acknowledgement}

Funded project: State Grid SGTYHT/19-JS-215

\section{References}

1. G.M. Qin, H.T. Fu, R. Xiao, Effect on DC Biasing on Main Transformers of Gezhouba Hydropower Station and Study on Restraining Measures, Water Power. J. 33(12), 79-81 (2007)

2. V.D. Albertson, J.M. Thorson, S.A. Miske, The effects of geomagnetic storms on electric power 
system, IEEE Transactions on power Apparatus and System. J. 93(4), 1031-1044 (1974)

3. D.Z. Kuai, C.M. Liu, Da. Wan, Experiment and Research of the Influence of Direct-current Magnetic Bias on Transformer, Jiangsu Electrical Engineering. J. 23(3), 1-5 (2004)

4. P.J. Boden, J.M. Evans, Reduction of stray-current attack in electrochemical machining, Electrochim. Acta. J. 16(7), 1071-1079 (1971)

5. W. Fan, The Numerical Simulation study on the Stray Current of Metro, DUT. D. (2012)

6. Y.B. Pang, Q.Z. Li, Discussions on Metro's Stray Current Model, JCIT(NSE). J. 21(11), 110-113,150 (2007)

7. J.W. Mei, G.S. Lin, Analysis of subway stray current under multi-train operation, Electric Railway. J. 28(4), 68-70 (2017)

8. K. Yu, Y.R. Ni, X. Zeng, Modeling and Analysis of Transformer DC Bias Current Caused by Metro Stray, IEEJ T Electr Electr. J. 15, 1507-1519 (2020)

9. J. Wang, Study on Metro Stray Current Distribution under Multi-train Operation ,SWJTU. D. (2012)

10. J.J. Wu, Transformer GIC DC bias magnetic treatment device and its optimization design ,NCEPU. D. (2012)

11. C.M. Liu, Mid-low Latitude Power Grid Geomagnetically Induced Currents and its Assessing Method,NCEPU. D. (2009) 